\title{
LA EVALUACIÓN FORMATIVA Y COMPARTIDA EN APRENDIZAJE- SERVICIO. UN EJEMPLO EN FORMACIÓN INICIAL DEL PROFESORADO
}

Formative and shared assessment in Learning-Service. An example in initial teacher education

A avaliação formativa e partilhada na Aprendizagem-Serviço. Um exemplo na formação inicial de professores

$\mathbf{M}^{\mathrm{a}}$ Luisa Santos Pastor (1)

L. Fernando Martínez Muñoz (2)

(1) Departamento de Educación Física, Deporte y Motricidad Humana. Facultad de Formación de Profesorado y Educación. Universidad Autónoma de Madrid. Teléfono: + 34 4973060. Correo electrónico: marisa.santos@,uam.es

(2) Departamento de Educación Física, Deporte y Motricidad Humana. Facultad de Formación de Profesorado y Educación. Universidad Autónoma de Madrid. Teléfono: + 34 4974486. Correo electrónico: $\underline{\text { f.martinez@uam.es }}$

\section{Resumen}

La evaluación formativa es clave para dar sentido al aprendizaje en los proyectos de Aprendizaje-Servicio. El seguimiento y orientación del docente permiten regular el aprendizaje y revisar los procesos que componen estas propuestas formativas. En este artículo se presentan algunas acciones que facilitan la evaluación formativa del proyecto de Aprendizaje-Servicio en la asignatura de Actividad Físico-Deportivas en el Medio Natural del Grado en Ciencias de la Actividad Física y el Deporte de la Universidad Autónoma de Madrid. Centraremos la mirada en los procesos reflexivos propuestos y en las herramientas construidas para hacer consciente al estudiante de su aprendizaje y ayudarle a comprender cómo puede mejorarlo. En concreto, se presentan los cuestionarios diseñados e implementados en cada fase del proyecto. Estas herramientas nos han ayudado a dar sentido al aprendizaje de los estudiantes y a mejorar la evaluación de los proyectos de Aprendizaje-Servicio, aunque debamos seguir revisándolos.

Palabras clave: Aprendizaje-Servicio; evaluación formativa; reflexión; cuestionarios 


\begin{abstract}
The assessment formative is key to make sense of the learning in the Service-Learning projects. The follow-up and guidance of the teacher allow to regulate learning and review the processes that make up these training proposals. This article presents some actions that facilitate the formative evaluation of the Service-Learning project in the subject of Physical-Sports Activity in the Natural Environment of the Degree in Physical Activity and Sports Sciences of the Autonomous University of Madrid. We will focus our attention on the reflexive processes proposed and on the tools built to make the student aware of his learning and help him to understand how he can improve it. Specifically, the questionnaires designed and implemented in each phase of the project are presented. These tools have helped us to make sense of the students' learning and to improve the evaluation of the Learning-Service projects, although we should continue to review them.
\end{abstract}

Keywords: Service- Learning; assessment formative; reflection; questionnaires

\title{
Resumo
}

A avaliação formativa é fundamental para dar sentido à aprendizagem nos projetos de Aprendizagem-Serviço. O acompanhamento e a orientação do professor permitem regular a aprendizagem e rever os processos que compõem estas propostas formativas. Neste artigo apresentam-se algumas ações que facilitam a avaliação formativa do projeto Aprendizagem-Serviço na disciplina de Atividade Físico-Desportiva no Meio Natural da Licenciatura em Ciências da Atividade Física e desporto da Universidade Autónoma de Madrid. Centraremos a nossa atenção nos processos reflexivos propostos e nas ferramentas construídas para tornar o aluno consciente da sua aprendizagem e ajudá-lo a compreender como pode melhorá-la. Apresentam-se em concreto, os questionários desenhados e implementados em cada fase do projeto. Essas ferramentas ajudaram-nos a dar sentido à aprendizagem dos alunos e a melhorar a avaliação dos projetos de Aprendizagem-Serviço, embora seja necessário continuar a analisá-los. Palavras-chave: Aprendizagem-Serviço-; avaliação formativa; reflexão; questionários 


\section{La evaluación formativa y compartida en Aprendizaje-Servicio. Un ejemplo en} formación inicial del profesorado

El Aprendizaje-Servicio (ApS) como enfoque o filosofía formativa reporta efectos positivos en la formación inicial del profesorado, teniendo una incidencia clara en el desarrollo de sus competencias profesionales y cívico-ciudadanas, como han constatado Rubio \& Escofet (2017).

La evaluación de los proyectos de $\mathrm{ApS}$ es fundamental para poder facilitar el aprendizaje de los estudiantes (Ruiz-Corbella \& García-Gutiérrez, 2018). En este sentido, la evaluación formativa nos ayuda a: recoger información de los avances del alumnado durante todo el proceso; hacerle consciente de sus aprendizajes y que reflexione sobre puntos fuertes y débiles; concretar qué y cómo mejorar; ofrecer un feedback; orientar sus tareas, evaluar las competencias específicas y transversales, etc.

La propuesta de evaluación formativa que exponemos a continuación, se está desarrollando en el proyecto de ApS de la asignatura Actividades Físico-deportivas en el Medio Natural del grado de CAFYD de la UAM. El objetivo se centra en que los estudiantes, mediante un trabajo en grupo, realicen un programa de actividad físicodeportiva en el medio natural con diferentes colectivos desfavorecidos. El proyecto consta de cuatro fases: diagnóstico de las necesidades del colectivo, diseño de la propuesta práctica, implementación y evaluación. En cada una de estas fases se proponen diferentes procedimientos para el seguimiento del aprendizaje, mediante un portafolio en el que se recopilan las evidencias grupales y personales del proceso (tabla 1).

Tabla 1.

Fases del proyecto de ApS y tareas de evaluación

\begin{tabular}{|c|c|}
\hline FASES PROYECTO & $\begin{array}{c}\text { PORTAFOLIO } \\
\text { TAREAS- REFLEXIONES }\end{array}$ \\
\hline $\begin{array}{l}\text { DIAGNÓSTICO } \\
\text { Conocen y valoran las necesidades que tiene el colectivo y qué } \\
\text { tienen que tener en cuenta para el diseño } \\
\text { DISEÑO }\end{array}$ & $\begin{array}{l}\text { CUESTIONARIOS PARA LA } \\
\text { REFLEXIÓN DE LOS } \\
\text { APRENDIZAJES EN CADA } \\
\text { FASE }\end{array}$ \\
\hline $\begin{array}{l}\text { Realizan un diseño práctica atendiendo a los contenidos de la } \\
\text { asignatura y a las necesidades/posibilidades del colectivo } \\
\text { IMPLEMENTACIÓN }\end{array}$ & $\begin{array}{c}\text { DIARIO PERSONAL DE } \\
\text { APRENDIZAJES }\end{array}$ \\
\hline $\begin{array}{l}\text { adecuación } \\
\text { EVALUACIÓN } \\
\text { Analizan el diseño y el desarrollo de la práctica y la replantean. }\end{array}$ & $\begin{array}{l}\text { INFORME COLECTIVO DE } \\
\text { APRENDIZAJES }\end{array}$ \\
\hline
\end{tabular}

La evaluación formativa y compartida en Aprendizaje-Servicio. Un ejemplo en formación inicial del profesorado 
La evaluación de los proyectos de ApS deben incorporar la reflexión en todo el proceso, siendo clave para lograr aprendizajes reales en los estudiantes. Para ello, es necesario diseñar herramientas que provoquen dicha reflexión en las diferentes fases del proyecto y garanticen un aprendizaje más significativo y profundo, haciéndoles conscientes del valor de los procesos, de sus repercusiones y de las dificultades que surgen. En esta línea, con el objetivo de favorecer los procesos de reflexión, se han diseñado y aplicado unos cuestionarios en Google Forms para cada una de las fases del proyecto y para las diferentes acciones vinculadas con el seguimiento y asesoramiento de estos proyectos, acomodando las preguntas al objetivo que se pretendía cubrir en cada una de las fases y acordes con el sentido que se proponía dar al aprendizaje. En la tabla 2 se concretan los ítems que componen cada cuestionario.

Tabla 2.

Cuestionarios para la reflexión del ApS

\begin{tabular}{|c|c|c|}
\hline $\begin{array}{c}\text { Cuestionario para la } \\
\text { Reflexión sobre... } \\
\end{array}$ & Fase & Ítems \\
\hline Ideas previas & Inicial & $\begin{array}{l}\text { - ¿Por qué has elegido hacer un proyecto de ApS? } \\
\text { ¿Cuáles son los compromisos personales que } \\
\text { adquieres en el proyecto? } \\
\text { - ¿Cuáles son tus expectativas ante el proyecto? } \\
\text { - ¿Qué crees que puedas aportar? }\end{array}$ \\
\hline $\begin{array}{l}\text { Necesidades/posibilidades } \\
\text { del colectivo }\end{array}$ & Diagnóstico & 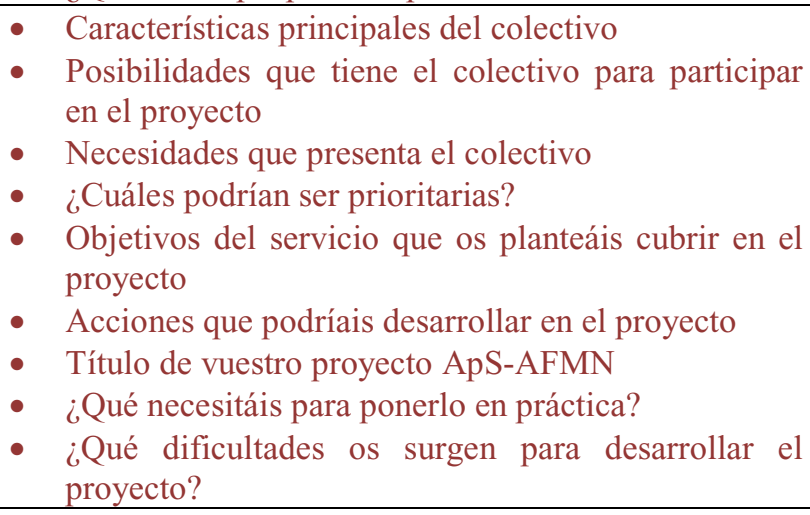 \\
\hline $\begin{array}{l}\text { Preparación de la sesión } \\
(*)\end{array}$ & Diseño & $\begin{array}{l}\text { - ¿Has tenido en cuenta las necesidades del colectivo } \\
\text { - ¿Cara hacer el diseño del proyecto de ApS-AFDMN? } \\
\text { - ¿Qué has tenido en cuenta? } \\
\text { - ¿Qué ha supuesto para ti preparar la intervención? } \\
\text { - ¿Qué dificultades te han surgido en esta fase? } \\
\text { - ¿Qué has las has solventado? } \\
\end{array}$ \\
\hline $\begin{array}{l}\text { Implementación de la } \\
\text { sesión }(*)\end{array}$ & Implementación & $\begin{array}{l}\text { - } \mathrm{N}^{\mathrm{o}} \text { de intervención del proyecto } \\
\text { - } \quad \text { Rol que has asumido } \\
\text { ¿En qué grado consideras que habéis conseguido los } \\
\text { objetivos propuestos? } \\
\text { - ¿Qué dificultades has tenido individualmente? } \\
\text { - ¿Qué dificultades han surgido en el grupo? } \\
\end{array}$ \\
\hline
\end{tabular}

La evaluación formativa y compartida en Aprendizaje-Servicio. Un ejemplo en formación inicial del profesorado 


\begin{tabular}{|c|c|c|}
\hline & & $\begin{array}{l}\text { - ¿Qué cambios realizarías? } \\
\text { - ¿Qué crees que has aportado al colectivo? } \\
\text { - Valora la calidad del servicio realizado (1 al 5) } \\
\text { - ¿Qué valoración darías a tu implicación en esta } \\
\text { sesión? (1 al 5) }\end{array}$ \\
\hline $\begin{array}{l}\text { Valoración de la sesión } \\
(*)\end{array}$ & Evaluación & $\begin{array}{l}\text { - } \text { Colectivo con el que has desarrollado el proyecto } \\
\text { - } \quad \text { Número de intervenciones realizadas } \\
\text { ¿Te ha parecido adecuado el número de sesiones } \\
\text { dedicado al desarrollo del proyecto? Justifica tu } \\
\text { respuesta } \\
\text { - ¿Qué es lo que más te ha ayudado a desarrollar el } \\
\text { proyecto? } \\
\text { - ¿Qué has aprendido? } \\
\text { - ¿Qué dificultades has tenido? } \\
\text { ¿Qué beneficios aporta a tu formación profesional y } \\
\text { personal? } \\
\text { ¿Qué consideras que has aportado a los receptores } \\
\text { del servicio? } \\
\text { ¿Qué ha sido lo más valioso de este proyecto? } \\
\text { Valora la aportación del proyecto de ApS a tu } \\
\text { formación profesional (1 al 5) } \\
\text { Valora la aportación del proyecto de ApS a tu } \\
\text { formación personal (1 al 5) }\end{array}$ \\
\hline Tutorías & Seguimiento & $\begin{array}{l}\text { - Motivo de la tutoría } \\
\text { - Tiempo aproximado que ha durado } \\
\text { - } \quad \text { Temas tratados en la tutoría } \\
\text { - ¿Qué has aportado a la mejora del proyecto? } \\
\text { - ¿Qué mejorarías? }\end{array}$ \\
\hline
\end{tabular}

(*) un cuestionario para cada propuesta de sesión.

Esta herramienta se aplica al finalizar cada fase, siendo enviadas a través del correo electrónico del estudiante. Deben ser completadas en un plazo corto, de modo que se pueda garantizar recordar lo sucedido, sin dejarlo en el olvido. En algunas ocasiones, las respuestas son el punto de partida para comenzar la siguiente fase del proceso, sirviendo de revisión de los aprendizajes.

\section{A modo de conclusión}

Para terminar, podemos indicar que la información recogida a través de esta herramienta favorece los procesos de reflexión y mejora de los aprendizajes del alumnado del grado. Como docentes nos ayuda a comprobar si los objetivos de aprendizaje previstos se han conseguido y en qué medida; así como a entender las necesidades de mejora de algunas de las acciones o procedimientos propuestos.

\section{Referencias}

Chiva-Bartoll, Ò., \& Gil Gómez, J. (Eds.) (2018). Aprendizaje-Servicio Universitario. Modelos de intervención e investigación en la formación inicial docente. 
Barcelona: Octaedro.

Rubio, L., \& Escofet, A. (Coords.) (2017). Aprendizaje-servicio (ApS): claves para su desarrollo en la universidad. Barcelona: Octaedro.

Ruiz-Corbella, M., \& García-Gutiérrez, J. (2019). Aprendizaje-Servicio. Los retos de la evaluación. Madrid: Narcea.

Uruñuela, P.M. (2018). Metodología del Aprendizaje-Servicio. Aprender mejorando el mundo. Madrid: Narcea.

La evaluación formativa y compartida en Aprendizaje-Servicio. Un ejemplo en formación inicial del profesorado 